\title{
IMPROVED TECHNIQUES FOR LOWER BOUNDS FOR ODD PERFECT NUMBERS
}

\author{
R. P. BRENT, G. L. COHEN, AND H. J. J. TE RIELE
}

\begin{abstract}
If $N$ is an odd perfect number, and $q^{k} \| N, q$ prime, $k$ even, then it is almost immediate that $N>q^{2 k}$. We prove here that, subject to certain conditions verifiable in polynomial time, in fact $N>q^{5 k / 2}$. Using this and related results, we are able to extend the computations in an earlier paper to show that $N>10^{300}$.
\end{abstract}

\section{INTRODUCTION}

A natural number $N$ is perfect if $\sigma(N)=2 N$, where $\sigma$ is the positive divisor sum function. It is not known whether odd perfect numbers exist. In an earlier paper [2], the first two authors described an algorithm for demonstrating that there is no odd perfect number less than a given bound $K$, and applied it with $K=10^{160}$.

That paper, and others referenced in it, are dependent on the simple observation that if $N$ is an odd perfect number and $q^{k} \| N$, where $q$ is prime and $k$ is even, then $N \geq q^{k} \sigma\left(q^{k}\right)>q^{2 k}$. Methods based on this observation require the explicit factorization of $\sigma\left(q^{k}\right)$ for large values of $q^{k}$, which imposes a practical limit on their effectiveness. Fewer factorizations would be required if it were known that $N>q^{l}$ for $l>2 k$. We shall prove below that, under certain conditions which are readily tested computationally and easily satisfied in the cases to be considered, we in fact have $N>q^{5 k / 2}$ (Theorem 2, below). In some cases the exponent on $q$ can be raised almost to $3 k$ (Theorem 3 ).

The main result of this paper (Theorem 1) is still heavily dependent on the algorithm in [2], and we assume familiarity with that paper. It was stated at the end of that work that to continue the algorithm to obtain any substantial improvement of the earlier result required the factorization of the 81-decimaldigit composite number $\sigma\left(13^{72}\right)$; this factorization has been completed and the result given in a postscript to [2]. But as our targeted lower bound increased, numerous other "unattainable" factorizations appeared to bar our way. One example is the factorization of $\sigma\left(3169^{36}\right)$, a composite number of 127 digits;

Received October 11, 1989; revised October 24, 1990.

1980 Mathematics Subject Classification (1985 Revision). Primary 11A25; Secondary 11Y05, $11 \mathrm{Y} 70$. 
since $3169^{5 \cdot 36 / 2}=3169^{90}>10^{315}$, this factorization could be avoided by application of Theorem 2. This approach was still not sufficient in some instances, but more powerful results along the same lines allowed us to avoid these factorizations as well.

We have thus been able to prove

Theorem 1. There is no odd perfect number less than $10^{300}$.

To prove Theorem 1 , there were nine cases, all detailed in $\S 4$, requiring special attention. Apart from these, the original algorithm of [2], with the " $q^{2 k}$ " result, was sufficient.

A preliminary version of the present work is contained in [3]. There, the lower bound $10^{200}$ was obtained.

To describe the new method, we need the definition below. For each $\alpha \geq 0$, $f_{\alpha}$ is a function defined on the positive integers and satisfying $1 \leq f_{\alpha}(n) \leq 2^{\alpha}$ (so in particular $f_{0}(n)=1$ ). We shall choose $f_{\alpha}$ as appropriate later.

Definition. Let $q$ be an odd prime and $k$ a positive integer. Define

$$
\begin{array}{r}
E_{\alpha}(q, k)=\left\{p^{\beta} \mid p \text { odd prime, } \beta \geq 2, \beta \text { even or } \beta \equiv p \equiv 1(\bmod 4),\right. \\
\left.(\exists j)\left(0<j \leq k, p^{\beta} / f_{\alpha}(p)<q^{2 j} \text { and } q^{j} \| \sigma\left(p^{\beta}\right)\right)\right\}
\end{array}
$$

and

$$
\varepsilon_{\alpha}(q, k)=\sum_{p^{\beta} \in E_{\alpha}(q, k)} \log _{q}\left(q^{2 j} f_{\alpha}(p) / p^{\beta}\right) .
$$

(The value of $j$ in the sum is that for which $q^{j} \| \sigma\left(p^{\beta}\right)$.)

Write $\varepsilon$ for $\varepsilon_{0}$.

We can compute an upper bound on $\varepsilon_{\alpha}(q, k)$ in time polynomial in $q$ and $k$ by an efficient "lifting" algorithm, described in Hardy and Wright [7, Theorem 123]. Usually, $\alpha=0$ and $\varepsilon(q, k)$ is quite small; numerical results will be given in $\S 3$.

We assume in the following that $N$ is an odd perfect number. According to Euler, we may write

$$
N=q^{k} \prod_{i=1}^{j} p_{i}^{\beta_{i}},
$$

where $q$ and the $p_{i}$ are distinct odd primes, $p_{1} \equiv \beta_{1} \equiv 1(\bmod 4)$ and $k \equiv$ $\beta_{2} \equiv \cdots \equiv \beta_{j} \equiv 0(\bmod 2)$. It is easy to show that $j \geq 2$, and we make implicit use of this below; in fact, it is known that $j \geq 7$ (Hagis [6]). Each $p_{i}^{\beta_{i}}$, and $q^{k}$, are called components of $N$.

Our new results follow.

Theorem 2. Let $N, q^{k}$ and $\varepsilon(q, k)$ be as above. Then, provided $k \geq 6 \varepsilon(q, k)$ and $\sigma\left(q^{k}\right)$ is not a square and has no prime factors less than $\frac{1}{2} q^{\varepsilon(q, k)}$, we have $N>q^{5 k / 2}$. 
Theorem 3. Let $N, q^{k}, \varepsilon(q, k)$ and $p_{1}$ be as above. Let $M$ be a unitary divisor of $N$ (that is, $M \mid N$ and $\operatorname{gcd}(M, N / M)=1$ ) such that $q \nmid M$, $q \nmid \sigma(M)$ and $p_{1} \nmid M$. Then

$$
N>\frac{1}{2} M q^{3 k-k_{1}-\varepsilon(q, k)},
$$

where $q^{k_{1}} \| p_{1}+1$.

\section{Proofs of Theorems 2 AND 3}

The proofs depend on a number of lemmas, some of which will also be used independently in the proof of Theorem 1.

Lemma 1. If $p$ and $q$ are odd primes with $p \mid \sigma\left(q^{k}\right)$ and $q^{m} \mid p+1$, then $k \geq 3 m$.

Proof. Since $q^{m} \mid p+1$, we have $p+1=2 a q^{m}$ for some $a>0$. Then, since $p \mid \sigma\left(q^{k}\right)=\left(q^{k+1}-1\right) /(q-1)$,

$$
q^{k+1}-1=\left(2 a q^{m}-1\right) R,
$$

where $R>0$, and this implies $k \geq m$. From the preceding equation, we have $R \equiv 1\left(\bmod q^{m}\right)$, so $R=b q^{m}+1$ say, and clearly $b>0$.

Thus,

$$
q^{k+1}-1=\left(2 a q^{m}-1\right)\left(b q^{m}+1\right),
$$

so $q^{k+1}>a q^{m} \cdot b q^{m} \geq q^{2 m}$, from which $k \geq 2 m$.

We also have

$$
q^{k+1-m}=2 a b q^{m}+2 a-b,
$$

so $b=2 a+\lambda q^{m}$, where $\lambda=2 a b-q^{k+1-2 m}$, the latter implying $\lambda \neq 0$. Then we cannot have both $b<q^{m}$ and $2 a<q^{m}$, since in that case

$$
|\lambda|=\frac{|b-2 a|}{q^{m}}<1
$$

a contradiction. Hence, $b \geq q^{m}$ or $2 a>q^{m}$.

From (1), if $2 a>q^{m}$, then

$$
q^{k+1}-1>\left(q^{2 m}-1\right)\left(q^{m}+1\right),
$$

so $q^{k+1}>q^{3 m}+q^{2 m}-q^{m} \geq q^{3 m}$; and if $b \geq q^{m}$, then

$$
q^{k+1}-1 \geq\left(2 q^{m}-1\right)\left(q^{2 m}+1\right),
$$

so $q^{k+1} \geq 2 q^{3 m}+2 q^{m}-q^{2 m}>q^{3 m}$. Either way, we infer that $k \geq 3 m$, as required.

The example $p=5, q=3, k=3, m=1$ shows this result to be best possible. 
Lemma 2. Let $q$ be an odd prime and let $S$ be any nonempty set of prime powers $p^{\beta}$, with $p$ odd and $\beta$ at least 2 and either even or satisfying $\beta \equiv p \equiv$ $1(\bmod 4)$. For each $p_{i}^{\beta_{i}} \in S$, suppose $q^{k_{i}} \| \sigma\left(p_{i}^{\beta_{i}}\right)$ and $k \geq \sum k_{i}$. Then

$$
\log _{q} \prod_{p_{i}^{\beta_{i}} \in S} \frac{\sigma\left(p_{i}^{\beta_{i}}\right)}{f_{\alpha}\left(p_{i}\right)}>2 \sum k_{i}-\varepsilon_{\alpha}(q, k) .
$$

Proof. We have quite generally that

$$
\frac{\sigma\left(p_{i}^{\beta_{i}}\right)}{f_{\alpha}\left(p_{i}\right)}>\frac{p_{i}^{\beta_{i}}}{f_{\alpha}\left(p_{i}\right)}=q^{2 k_{i}-\left(2 k_{i}-\log _{q}\left(p_{i}^{\beta_{i}} / f_{\alpha}\left(p_{i}\right)\right)\right)}=q^{2 k_{i}-\log _{q}\left(q^{2 k_{i}} f_{\alpha}\left(p_{i}\right) / p_{i}^{\beta_{i}}\right)},
$$

while if $p_{i}^{\beta_{i}} \in S \backslash E_{\alpha}(q, k)$, then $p_{i}^{\beta_{i}} / f_{\alpha}\left(p_{i}\right) \geq q^{2 k_{i}}$. Thus, where $E_{\alpha}=E_{\alpha}(q, k)$,

$$
\begin{aligned}
\log _{q} \prod_{p_{i}^{\beta_{i}} \in S} \frac{\sigma\left(p_{i}^{\beta_{i}}\right)}{f_{\alpha}\left(p_{i}\right)} & >2 \sum k_{i}-\sum_{p_{i}^{\beta_{i}} \in S \cap E_{\alpha}} \log _{q}\left(q^{2 k_{i}} f_{\alpha}\left(p_{i}\right) / p_{i}^{\beta_{i}}\right) \\
& \geq 2 \sum k_{i}-\sum_{p_{i}^{\beta_{i}} \in E_{\alpha}} \log _{q}\left(q^{2 k_{i}} f_{\alpha}\left(p_{i}\right) / p_{i}^{\beta_{i}}\right) \\
& \geq 2 \sum k_{i}-\varepsilon_{\alpha}(q, k)
\end{aligned}
$$

as required.

We remark that Lemmas 1 and 2 require no reference to odd perfect numbers.

Lemma 3. Let $N, q^{k}$ and $p_{1}^{\beta_{1}}$ be as in $\S 1$.

(i) If $\beta_{1}>1$, then

$$
N>\frac{1}{2} q^{3 k-\varepsilon_{\alpha}(q, k)} \prod_{i=1}^{j} f_{\alpha}\left(p_{i}\right)
$$

(ii) If $\beta_{1}=1$, then

$$
N>q^{3 k-k_{1}-\varepsilon_{\alpha}(q, k)} \prod_{i=2}^{j} f_{\alpha}\left(p_{i}\right),
$$

where $q^{k_{1}} \| p_{1}+1$.

Proof. (i) Apply Lemma 2 with $S$ equal to the set of components of $N / q^{k}$. Then, in Lemma 2, $\sum k_{i}=k$ and

$$
\begin{aligned}
2 N=\sigma(N) & =\sigma\left(q^{k}\right) \prod_{i=1}^{j} \frac{\sigma\left(p_{i}^{\beta_{i}}\right)}{f_{\alpha}\left(p_{i}\right)} \prod_{i=1}^{j} f_{\alpha}\left(p_{i}\right) \\
& >q^{k} \cdot q^{2 k-\varepsilon_{a}(q, k)} \cdot \prod_{i=1}^{j} f_{\alpha}\left(p_{i}\right) .
\end{aligned}
$$


(ii) We again apply Lemma 2, this time with $S$ equal to the set of components of $N / q^{k} p_{1}$. Then $\sum k_{i}=k-k_{1}$ and

$$
\begin{aligned}
2 N=\sigma(N) & =\sigma\left(q^{k}\right) \sigma\left(p_{1}\right) \prod_{i=2}^{j} \frac{\sigma\left(p_{i}^{\beta_{i}}\right)}{f_{\alpha}\left(p_{i}\right)} \prod_{i=2}^{j} f_{\alpha}\left(p_{i}\right) \\
& >q^{k} \cdot\left(p_{1}+1\right) \cdot q^{2\left(k-k_{1}\right)-\varepsilon_{\alpha}(q, k)} \cdot \prod_{i=2}^{j} f_{\alpha}\left(p_{i}\right) .
\end{aligned}
$$

Since $p_{1}+1 \geq 2 q^{k_{1}}$, the result follows.

Corollary 1. Let $N, q^{k}$ and $p_{1}^{\beta_{1}}$ be as above. If either (i) $\beta_{1}>1$, or (ii) $\beta_{1}=1$ and $p_{1} \mid \sigma\left(q^{k}\right)$, then

$$
N>q^{8 k / 3-\varepsilon(q, k)} \text {. }
$$

Proof. Take $\alpha=0$ in Lemma 3. (i) Since $k \geq 2$ and $q \geq 3$, we have $q^{k / 3}>2$ and the result follows from Lemma 3 (i). (ii) From Lemma $1, k \geq 3 k_{1}$, so the result follows from Lemma 3 (ii) since $3 k-k_{1} \geq 3 k-k / 3=8 k / 3$.

Lemma 4. Let $N, q^{k}$ and $p_{1}$ be as in $\S 1$. Suppose that $\sigma\left(q^{k}\right)$ is not a perfect square and is not divisible by $p_{1}$ or any prime number less than $B$. Then

$$
N^{2}>2 B q^{5 k-\varepsilon_{\alpha}(q, k)} \prod_{i=2}^{j} f_{\alpha}\left(p_{i}\right) .
$$

Proof. We shall prove this lemma in the case where $q \mid p_{1}+1$ and $p_{1} \| N$. (Minor adjustments are needed for the other cases.) Suppose $q^{k_{1}} \| p_{1}+1$. Since $\sigma\left(q^{k}\right)$ is not a perfect square, there is a prime, $p_{2}$ say, but not $p_{1}$, which divides $\sigma\left(q^{k}\right)$ to an odd power and so divides $N$ to a higher (even) power. Also $p_{1}+1 \geq 2 q^{k_{1}}$ and $p_{2} \geq B$, so

$$
\begin{aligned}
N & \geq q^{k} \sigma\left(q^{k}\right) p_{1} p_{2} \\
& \geq q^{k} \cdot q^{k}\left(1+q^{-1}\right) \cdot 2 q^{k_{1}}\left(1-\frac{1}{2} q^{-k_{1}}\right) \cdot B \\
& >2 B q^{2 k+k_{1}} .
\end{aligned}
$$

From Lemma 3 (ii), we also have $N>q^{3 k-k_{1}-\varepsilon_{\alpha}(q, k)} \prod_{i=2}^{j} f_{\alpha}\left(p_{i}\right)$. Hence

$$
N^{2}>2 B q^{5 k-\varepsilon_{\alpha}(q, k)} \prod_{i=2}^{j} f_{\alpha}\left(p_{i}\right)
$$

as required.

Proof of Theorem 2. Take $\alpha=0$. Since $k \geq 6 \varepsilon(q, k)$, we have $8 k / 3-$ $\varepsilon(q, k) \geq 5 k / 2$, and the theorem follows from Corollary 1 , unless $\beta_{1}=1$ and $\sigma\left(q^{k}\right)$ is not divisible by $p_{1}$. But then the result follows from Lemma 4 , with $B \geq \frac{1}{2} q^{\varepsilon(q, k)}$. 
Proof of Theorem 3. As in the proof of Lemma 3, we consider two cases. If $\beta_{1}>1$, then apply Lemma 2 with $S$ equal to the set of components of $N / M q^{k}$. Then $\sum k_{i}=k$, since $q \nmid \sigma(M)$, and

$$
\begin{aligned}
2 N=\sigma(N)=\sigma(M) \sigma\left(\frac{N}{M}\right) & =\sigma(M) \sigma\left(q^{k}\right) \prod_{\substack{i=1 \\
p_{i} \nmid M \\
j}}^{j} \sigma\left(p_{i}^{\beta_{i}}\right) \\
& >M \cdot q^{k} \cdot q^{2 k-\varepsilon(q, k)}=M q^{3 k-\varepsilon(q, k)} .
\end{aligned}
$$

If $\beta_{1}=1$, then apply Lemma 2 with $S$ equal to the set of components of $N / M q^{k} p_{1}$. Then $\sum k_{i}=k-k_{1}$ and

$$
\begin{aligned}
2 N & =\sigma(M) \sigma\left(q^{k}\right) \sigma\left(p_{1}\right) \prod_{\substack{i=2 \\
p_{i}+M \\
j}}^{j} \sigma\left(p_{i}^{\beta_{i}}\right) \\
& >M \cdot q^{k} \cdot 2 q^{k_{1}} \cdot q^{2\left(k-k_{1}\right)-\varepsilon(q, k)}=2 M q^{3 k-k_{1}-\varepsilon(q, k)} .
\end{aligned}
$$

The result follows.

\section{Computation of $\varepsilon_{\alpha}(q, k)$}

Theorem 2 is useful because an upper bound on $\varepsilon_{\alpha}(q, k)$ can be computed in time which is bounded by a low-degree polynomial in $q$ and $k$. We first outline an algorithm for this computation, and then give a numerical example.

Suppose $p^{\beta} \in E_{\alpha}(q, k)$, where $E_{\alpha}(q, k)$ is defined in $\S 1$. Since $p \geq 3$ and $p^{\beta}<2^{\alpha} q^{2 k}$, we have $\beta<\alpha+2 k \log _{3} q$. Thus, to establish the polynomial-time result, it is sufficient to suppose that $\beta$ is fixed.

Define $F(x)=1+x+x^{2}+\cdots+x^{\beta}$. We can enumerate the set $S_{j}$ of least positive residues modulo $q^{j}$ of $F(x) \equiv 0\left(\bmod q^{j}\right)$ by the "lifting" algorithm described in [7, §8.3]. If $j=1$, we can simply check all possible solutions $1,2, \ldots, q-1$ (although faster methods, using a primitive root $(\bmod q)$, are preferable if $q$ is large). If $j>1$, we apply Theorem 123 of [7] to obtain $S_{j}$ from $S_{j-1}$, using what is essentially an application of Newton's method. Since $F(x)$ is a polynomial of degree $\beta$, the number $\left|S_{j}\right|$ of solutions is bounded by $\beta$.

Define $T_{j}=\left\{s+\lambda q^{j} \mid s \in S_{j}, \lambda \geq 0,\left(s+\lambda q^{j}\right)^{\beta}<f_{\alpha}\left(s+\lambda q^{j}\right) q^{2 j}\right\}$. Clearly, $\left|T_{j}\right| \leq\left\lceil 2^{\alpha / \beta}\right\rceil\left|S_{j}\right|$. Since $p^{\beta} \in E_{\alpha}(q, k)$, there is some $j, 0<j \leq k$, such that $q^{j} \| \sigma\left(p^{\beta}\right)$ and $p^{\beta}<f_{\alpha}(p) q^{2 j}$. Thus, to enumerate such $p^{\beta}$, we need only check the elements of $T_{1}, T_{2}, \ldots, T_{k}$ for primality. In order to obtain an upper bound on $\varepsilon_{\alpha}(q, k)$, it is sufficient to use a polynomial-time probabilistic primality test, for the inclusion of a composite $p$ will only increase the computed sum $\sum \log _{q}\left(q^{2 j} f_{\alpha}(p) / p^{\beta}\right)$. In practice, below, the upper bounds on each $\varepsilon_{\alpha}(q, k)$ are in fact exact results, rounded up if not zero, since each $p$ used was shown to be prime. 
Example. To illustrate the algorithm, consider the computation of $\varepsilon(3169,36)$. If $\beta=2$, then $F(x)=1+x+x^{2}$, so $S_{1}=\{97,3071\}$ is the set of solutions of $F(x) \equiv 0(\bmod 3169)$. We construct the sets $S_{2}, S_{3}, \ldots, S_{36}$ as in [7, §8.3], and in each case $\left|S_{j}\right|=2$. (In this example, $\alpha=0$, so $T_{j}=S_{j}$; in general, we would add a small number of multiples of $q^{j}$ to the elements of $S_{j}$ in order to obtain the set $T_{j}$.) Applying a probabilistic primality test to the elements of $T_{1}, T_{2}, \ldots, T_{36}$ rules out all but three possible odd prime $p$ such that $3169^{j} \| \sigma\left(p^{2}\right)$ :

$$
\begin{aligned}
& j=1, \quad p=97 \\
& j=3, \quad p=5875516237 ; \text { and } \\
& j=11, \quad p=266602399893630549086712579594816998201 .
\end{aligned}
$$

The contributions $\log _{3169}\left(3169^{2 j} / p^{2}\right)$ from these three pairs $(j, p)$ are respectively bounded above by $0.8651,0.4192$, and 0.0482 .

For $\beta \geq 4$, we proceed similarly, but we find no solutions satisfying all the constraints. (This is typical, since it is unlikely that the constraint $p^{\beta}<f_{\alpha}(p) q^{2 j}$ will be satisfied if $\beta \geq 4$.) We conclude that only the three pairs given above can contribute to $\varepsilon(3169,36)$, so $\varepsilon(3169,36) \leq 1.3325$.

Table 1 gives the details of all nonzero contributions to $\varepsilon_{\alpha}(q, k)$ in the cases relevant to the proof of Theorem 1 . Note that there is only one case with $\beta>2$.

\section{Proof of Theorem 1}

Except for the nine cases to be discussed below, the proof is a straightforward extension of the algorithmic method given in [2]. In particular, it is still valid that the theorem will follow once the primes $127,19,7,11,31,13,3$, and 5 are eliminated as possible divisors of $N$. (As in [2], the elimination of these primes was carried out in the order given.)

The computer output towards the proof of Theorem 1 has 12655 lines. Some relevant extracts are shown below. In these, D means the indicated divisor has already been considered; for details, see [2]. The cases requiring special attention are the following.

(i) Line 7343 concerns the possibility that $3221^{42} \| N$. Note that $\sigma\left(3221^{42}\right)$ $=c_{148}$, a composite number with 148 decimal digits, unable to be factorized at this time. We have $\varepsilon(3221,42)=\log _{3221}\left(3221^{2} / 11^{4}\right) \leq 0.8126$ (see Table 1); the conditions of Theorem 2 are satisfied, so

$$
N>3221^{105}>10^{368} \text {. }
$$

(Such discussions will subsequently be much abbreviated.)

(ii) Line $7163, \sigma\left(7^{172}\right)=c_{146}, \varepsilon(7,172) \leq 4.1400$. By Theorem 2 ,

$$
N>7^{430}>10^{363} \text {. }
$$




\section{TABLE 1}

Nonzero contributions to $\varepsilon_{\alpha}(q, k), q^{j} \| \sigma\left(p^{\beta}\right)$

\begin{tabular}{|c|c|c|c|c|c|c|}
\hline$\alpha$ & $q$ & $k$ & $j$ & $\beta$ & $\log _{q}\left(q^{2 j} f_{\alpha}(p) / p^{\beta}\right)$ & $p$ (see below) \\
\hline 0 & 7 & 172 & $\begin{array}{r}8 \\
19 \\
25 \\
61 \\
119 \\
150\end{array}$ & $\begin{array}{l}2 \\
2 \\
2 \\
2 \\
2 \\
2\end{array}$ & 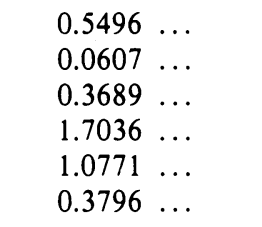 & $\begin{array}{l}3376853 \\
10744682090246617 \\
936579478224094047977 \\
6778 \ldots \\
1292 \ldots \\
4020 \ldots\end{array}$ \\
\hline 0 & 3221 & 42 & 1 & 4 & $0.8125 \ldots$ & 11 \\
\hline 0 & 612067 & 22 & $\begin{array}{r}1 \\
17\end{array}$ & $\begin{array}{l}2 \\
2\end{array}$ & $\begin{array}{l}0.3398 \ldots \\
0.2253 \ldots\end{array}$ & $\begin{array}{l}63601 \\
5291 \ldots\end{array}$ \\
\hline 0 & 3169 & 36 & $\begin{array}{r}1 \\
3 \\
11\end{array}$ & $\begin{array}{l}2 \\
2 \\
2\end{array}$ & $\begin{array}{ll}0.8650 & \ldots \\
0.4191 & \ldots \\
0.0481 & \ldots\end{array}$ & $\begin{array}{l}97 \\
5875516237 \\
2666 \ldots\end{array}$ \\
\hline \multicolumn{7}{|c|}{ Large primes $p$ occurring above } \\
\hline \multicolumn{7}{|c|}{677822686658425215407071060694728215733634249273881} \\
\hline \multicolumn{7}{|c|}{$\begin{array}{c}1292705670586158487568763039916765694399830534390031 \\
9630427909162956962804028786608263511417448696587\end{array}$} \\
\hline \multicolumn{7}{|c|}{$\begin{array}{c}40205637668275700485119982296550937753326347643003642516228059494 \\
71752457774955475739650336310509313359098137765181615640612833\end{array}$} \\
\hline \multicolumn{7}{|c|}{$\begin{array}{r}52915283185303600217046163660913353511950087333134 \\
106429889256916343584191707324489815681786937851\end{array}$} \\
\hline \multicolumn{7}{|c|}{2666023998936305490867125795948169988201} \\
\hline
\end{tabular}

(iii) Line $7985, \sigma\left(612067^{22}\right)=c_{128}, \varepsilon(612067,22) \leq 0.5652$. By Theorem 2,

$$
N>612067^{55}>10^{318} \text {. }
$$

(iv) Line $8866, \sigma\left(3169^{36}\right)=c_{127}, \varepsilon(3169,36) \leq 1.3324$. By Theorem 2 ,

$$
N>3169^{90}>10^{315} \text {. }
$$

(v) Line $4479, \sigma\left(467^{46}\right)=c_{123}$. We apply Lemma 3, with $\alpha=0$. The antecedents of this case (see Figure 1) show that $p_{1}=2801$ and $k_{1}=1$. Since $\varepsilon(467,46)=0$,

$$
N>467^{137}>10^{365} \text {. }
$$

FIGURE 1. $\sigma\left(467^{46}\right)$

\begin{tabular}{cc}
\hline $4340:$ & $7^{2} \Rightarrow 19 \ldots, \quad$ D \\
$4341:$ & $7^{4} \Rightarrow 2801$ \\
$4342:$ & $2801^{1} \Rightarrow 3.467$ \\
$4343:$ & $467^{2} \Rightarrow 19 \ldots, \quad$ D \\
& $($ some lines omitted) \\
$4479:$ & $467^{46} \Rightarrow c_{123}, \quad$ case $(v)$ \\
\hline
\end{tabular}


FigURE 2. $\sigma\left(191^{42}\right)$ and $\sigma\left(191^{46}\right)$

\begin{tabular}{ll}
\hline $8653:$ & $13^{1} \Rightarrow 7, \quad \mathrm{D}$ \\
$8654:$ & $\begin{array}{l}13^{2} \Rightarrow 3 \cdot 61 \\
(\text { some lines omitted })\end{array}$ \\
$9473:$ & $13^{4} \Rightarrow 30941$ \\
$9474:$ & $30941^{1} \Rightarrow 3^{4} \cdot 191$ \\
$9475:$ & $191^{2} \Rightarrow 7 \ldots, \quad \mathrm{D}$ \\
& $($ some lines omitted $)$ \\
$9526:$ & $191^{42} \Rightarrow c_{96}, \quad$ case (viii) \\
$9527:$ & $191^{46} \Rightarrow c_{105}, \quad$ case (vi) \\
\hline
\end{tabular}

(vi) Line 9527, $\sigma\left(191^{46}\right)=c_{105}$. See Figure 2. Lemma 3, with $\alpha=0$, $p_{1}=30941, k_{1}=1$ and $\varepsilon(191,46)=0$, gives

$$
N>191^{137}>10^{312} \text {. }
$$

(vii) Line 11343, $\sigma\left(36389^{22}\right)=c_{101}$. Observing the antecedents of this case (Figure 3), we may apply Theorem 3 with $M=3^{\beta_{2}} \geq 3^{18}, p_{1}=363889$, $k_{1}=1$ and $\varepsilon(36389,22)=0$. (Since 3 is a primitive root $(\bmod 36389)$ and $\beta_{2}$ is even, we know that $36389+\sigma\left(3^{\beta_{2}}\right)$.) Then

$$
N>\frac{1}{2} \cdot 3^{18} 36389^{65}>10^{304} \text {. }
$$

(viii) Line 9526, $\sigma\left(191^{42}\right)=c_{96}$. The antecedents of this case (Figure 2) show that $p_{1}=30941$ and $191 \| \sigma\left(p_{1}\right)$. Then, in Lemma $3, k_{1}=1$ and $f_{\alpha}\left(p_{1}\right) \geq 1$, so that

$$
N>q^{3 k-1-\varepsilon_{\alpha}(q, k)} \prod_{i=2}^{j} f_{\alpha}\left(p_{i}\right),
$$

with $q=191, k=42$. The bound obtainable from Lemma 3 with $\alpha=0$ is not quite good enough, so (for the first time) we need to take $\alpha>0$. For any prime $p$, we set

$$
f_{\alpha}(p)=\left\{\begin{array}{cl}
\left(\frac{p}{p-1}\right)^{\alpha} & \text { if } p \mid N, p \neq 191 \text { or } 30941 \\
1 & \text { otherwise. }
\end{array}\right.
$$

Since

$$
2=\frac{\sigma(N)}{N}<\prod_{p \mid N} \frac{p}{p-1}=\frac{191}{190} \frac{30941}{30940} \prod_{i=2}^{j} \frac{p_{i}}{p_{i}-1}
$$

we have

$$
\prod_{i=2}^{j} f_{\alpha}\left(p_{i}\right)>\left(2 \cdot \frac{190}{191} \cdot \frac{30940}{30941}\right)^{\alpha}
$$


We need to take $\alpha$ large enough that $\prod_{i=2}^{j} f_{\alpha}\left(p_{i}\right)$ is large but not so large that $\varepsilon_{\alpha}(191,42)>0$. After some experimentation, we find that with $\alpha=50$ we have $\varepsilon_{50}(191,42)=0$ and

$$
N>191^{125}\left(2 \cdot \frac{190}{191} \cdot \frac{30940}{30941}\right)^{50}>10^{300} .
$$

FIGURE 3. $\sigma\left(36389^{22}\right)$

\begin{tabular}{cc}
\hline $10615:$ & $\begin{array}{l}3^{2} \Rightarrow 13, \quad \mathrm{D} \\
\text { (some lines omitted) }\end{array}$ \\
$11214:$ & $3^{18} \Rightarrow 1597 \cdot 363889$ \\
$11215:$ & $363889^{1} \Rightarrow 5 \cdot 36389$ \\
$11216:$ & $36389^{2} \Rightarrow 1429 \cdot 926659$ \\
& $($ some lines omitted $)$ \\
$11343:$ & $36389^{22} \Rightarrow c_{101}, \quad$ case (vii) \\
\hline
\end{tabular}

(ix) Line 12201, $\sigma\left(3^{240}\right)=c_{115}$. Since $\varepsilon(3,240)=0$ and

$$
3^{8 \cdot 240 / 3}=3^{640}>10^{305}
$$

by Corollary 1 we may assume that $\beta_{1}=1$ and $p_{1} \nmid \sigma\left(3^{240}\right)$. Then, from Lemma 3 (ii),

$$
N>3^{720-k_{1}}>10^{300}
$$

if $k_{1} \leq 91$. Thus we may assume further that $k_{1} \geq 92$, so that $p_{1}>3^{92}$. For any prime $p$, we set

$$
f_{\alpha}(p)=\left\{\begin{array}{cl}
\left(\frac{p}{p-1}\right)^{\alpha} & \text { if } p \mid N, p \neq 3 \text { or } p_{1}, \\
1 & \text { otherwise. }
\end{array}\right.
$$

Then, as in (viii),

$$
\prod_{i=2}^{j} f_{\alpha}\left(p_{i}\right)>\left(2 \cdot \frac{2}{3} \cdot \frac{p_{1}-1}{p_{1}}\right)^{\alpha} .
$$

We find that with $\alpha=221$ we have $\varepsilon_{221}(3,240) \leq 0.9381$. (It is relevant for this calculation that $7,13,19,31$ are "forbidden" divisors. Otherwise, there would be contributions from these primes to the sum defining $\varepsilon_{221}(3,240)$.) Using Lemma 4 with $B=1$, we have

$$
N^{2}>2 \cdot 3^{1200-0.9381}\left(2 \cdot \frac{2}{3} \cdot \frac{3^{92}-1}{3^{92}}\right)^{221}>10^{600}
$$

This completes the proof of Theorem 1 .

The entire computer output is printed in [4]. A copy has been deposited in the UMT file of this journal. 
We should mention that the size of the task precluded us from being as fastidious as we were in [2]. The proof contains 199 partial factorizations. We estimate that at most 1658 lines would have been saved if those factorizations were completed. In most cases, the extra lines result from expanding the proof tree using a smaller prime than would be available if the complete factorization at the relevant line were known. In ten cases, the program had to backtrack from an assumed prime divisor $q$ to another in the same or an earlier branch in order to avoid a composite $\sigma\left(q^{k}\right)$ for which we could find no useful factors. The nine special cases (i)-(ix) arose because this option was not available or was impractical. (This can be seen in Figures 1 and 2.) On the other hand, there were three cases where the proof was shortened by branching on a prime smaller than the largest available (but there was no systematic search for such possibilities).

The program for the proof of Theorem 1 differs from that used in [2] in that, besides calculating bounds named B1, B2 and B3 there, it also calculates, when necessary, "bounds" named B25 and B30, which are $\left\lfloor\log _{10} q^{5 k / 2}\right\rfloor$ and $\left\lfloor\log _{10} q^{3 k}\right\rfloor$, respectively, for the current assumed component $q^{k}$. However, the B25 and B30 "bounds" need not exceed $10^{300}$, and the program does not incorporate the calculation of values of $\varepsilon_{\alpha}$, so that they are not rigorous and are used only to flag the need for special discussions, such as those above.

A supplement published with [2] contained the output for that proof. In [4], we include a list of factors of $p^{n}-1$ for $p$ prime, $13 \leq p<10000$, and those values of $n$ (all prime) which arose in our work. This complements the lists in [5] and should prove similarly useful. We take this opportunity to announce the availability of a machine-readable database of factors [1], including all those necessary for the proof of Theorem 1 .

\section{ACKNOWLEDGMENTS}

Some of the factorizations required for the proof of Theorem 1 were obtained using the Fujitsu VP-100 of the Australian National University Supercomputer Facility. Some others were obtained from [5]. We thank the referee for assistance in improving the exposition.

\section{BIBLIOGRAPHY}

1. R. P. Brent, Factor: an integer factorization program for the IBM PC, Report TR-CS-89-23, Computer Sciences Laboratory, Australian National University, October 1989.

2. R. P. Brent and G. L. Cohen, A new lower bound for odd perfect numbers, Math. Comp. 53 (1989), 431-437, S7-S24.

3. R. P. Brent, G. L. Cohen, and H. J. J. te Riele, An improved technique for lower bounds for odd perfect numbers, Report TR-CS-88-08, Computer Sciences Laboratory, Australian National University, August 1988.

4. R. P. Brent, G. L. Cohen, and H. J. J. te Riele, Improved techniques for lower bounds for odd perfect numbers, Report CMA-R50-89, Centre for Mathematical Analysis, Australian National University, October 1989. 
5. J. Brillhart, D. H. Lehmer, J. L. Selfridge, B. Tuckerman, and S. S. Wagstaff, Jr., Factorizations of $b^{n} \pm 1, b=2,3,5,6,7,10,11,12$ up to high powers, 2 nd ed., Contemp. Math., vol. 22, Amer. Math. Soc., Providence, RI, 1988.

6. P. Hagis, Jr., Outline of a proof that every odd perfect number has at least eight prime factors, Math. Comp. 34 (1980), 1027-1032.

7. G. H. Hardy and E. M. Wright, An introduction to the theory of numbers, 4th ed., Oxford Univ. Press, 1962.

Computer Sciences laboratory, Research School of Physical Sciences, The Australian National University, GPO Box 4, Canberra, ACT 2601, Australia

E-mail address: rpb@cslab.anu.edu.au

School of Mathematical Sciences, University of Technology, Sydney, PO Box 123 , BROADWAY, NSW 2007, Australia

Centrum voor Wiskunde en Informatica, Kruislaan 413, 1098 SJ Amsterdam, The NETHERLANDS 